\title{
EL ROL DE LOS GOBIERNOS REGIONALES Y LOCALES EN EL PERÚ PARA IMPULSAR EL ACUERDO DE PARÍS
}

\author{
Alfredo Ladrón de Guevara \\ Cámara Peruana de Energías Renovables (CAMPER)
}

\section{Introducción}

Estas reflexiones buscan poner en agenda la importancia que tienen los gobiernos locales y regionales en el cumplimiento del Acuerdo de París, las mismas que surgieron de las opiniones vertidas en un foro llamado «Gobiernos Azules para impulsar el Acuerdo de París en el Perú» (fines de octubre del 2018), organizado por la Cámara Peruana de Energías Renovables y el Instituto de Ciencias por la Naturaleza, Territorio y Energías Renovables de la Pontificia Universidad Católica del Perú.

En dicho evento se definió como Gobiernos Azules a aquellos gobiernos que estuvieran comprometidos con la lucha contra el Cambio Climático de acuerdo con los lineamientos de disminución en la emisión de gases de efecto invernadero propuestas en el mencionado Acuerdo, y se lo utilizó simplemente porque consideramos que es un nombre que genera una mayor conciencia respecto de una problemática que es común a todo el planeta, el mismo que en perspectiva tiene un color azul.

Al respecto, en dicho Foro se escucharon las ponencias de diferentes funcionarios del Ministerio del Ambiente, del Ministerio de Energía y Minas y de la Municipalidad de Lima que sirvieron como introducción a un taller en donde diferentes representantes de la sociedad civil respondieron a dos preguntas: 1 . ¿cuáles son las limitaciones que tienen los gobiernos subnacionales para implementar el Acuerdo de París? y 2 ¿cuáles son las propuestas de solución? 


\section{Limitaciones de los gobiernos subnacionales}

Con respecto a las limitaciones, los participantes de la sociedad civil llegaron a las siguientes conclusiones:

Tabla 1: Limitaciones de los gobiernos subnacionales para implementar el Acuerdo de París

\begin{tabular}{ll}
\hline \multirow{2}{*}{ Primer grupo } & Desconocimiento y desidia de las autoridades. \\
- Falta de profesionales capacitados. \\
- Resistencia al cambio y dificultad para adaptarse a las nuevas \\
tecnologías por parte de la población.
\end{tabular}

$\mathrm{Al}$ respecto podemos agrupar en tres las limitaciones principales para el crecimiento de gobiernos azules: ignorancia, desidia y corrupción.

En cuanto a la ignorancia, podríamos sintetizar los aportes en: falta de conocimiento y entendimiento de la problemática por parte de alcaldes y gobernadores; falta de profesionales y cuadros técnicos capacitados para el debido proceso de desarrollo, supervisión y control del monitoreo ambiental; falta de difusión; desconocimiento de las herramientas financieras y falta de educación ambiental.

Al respecto consideramos que la ignorancia se debe a la falta de conocimiento y entendimiento de las repercusiones del cambio climático, no sólo para el mundo sino también para sus respectivas circunscripciones políticas y sociales. Muchos lo ven también como algo lejano y que no los va a perjudicar.

Dicha falta de conocimiento y entendimiento se evidencia en la ausencia de capacitación y de información suficiente sobre la problemática, consecuencias y sus posibles soluciones, tanto a nivel de las autoridades políticas, de los 
funcionarios, técnicos y de la ciudadanía. No hay capacitación a los profesores ni a los funcionarios, y menos a los políticos en esta materia. Por ello, falta visión política para entender que la lucha contra el cambio climático es real, actual y transversal a toda actividad pública y privada, y que son acciones conjuntas y de largo aliento las que se deben ejecutar.

La transmisión de conocimiento en capacitaciones, mediciones, proyectos, asesorías y estímulos requiere de un presupuesto especial, el mismo que en nuestro país principalmente se concentra en el Ministerio del Ambiente, que es el que menos presupuesto maneja en comparación con los demás ministerios.

Por último, en cuanto a la falta de conocimiento de las herramientas financieras mencionada, si bien existen iniciativas públicas y privadas, éstas parecen no tener la fuerza, el impacto o el apoyo comunicacional suficiente como para que se tenga presente en el ideario de la ciudadanía.

En cuanto a la desidia o falta de interés, podríamos sintetizar los aportes en: desidia de las autoridades; resistencia al cambio y dificultad para adaptarse a nuevas tecnologías por parte de la población; falta de presupuesto y de gestión eficiente en el manejo económico y desinterés por diferentes prioridades políticas (como seguridad, pobreza).

Al respecto consideramos que el desinterés podría seguir existiendo aun cuando el sector público y el privado estuvieran informados y entendieran de las repercusiones del cambio climático -tanto a nivel ambiental, humano y económico-, mientras no existan ganancias o pérdidas, políticas o económicas de corto plazo.

Por ejemplo, en el caso de la energía, la existencia de petróleo, gas y de energía hidroeléctrica impiden que se vea como prioritaria la seguridad climática y energética como motor de una transición energética, aun cuando las fuentes de energía mencionadas vayan a acabarse o a verse afectadas por sequias, producto del cambio climático en el futuro cercano.

Por otro lado, el desconocimiento de herramientas financieras, hace que no se impulse la conciencia y el interés nacional al respecto. Sobre ello, hay que ser claros en cuanto al sector privado; ellos esperan el beneficio económico para invertir, y eso falta fomentar sin esperar a que los precios lleguen a ser competitivos por sí mismos, por el particular impacto que el cambio climático tendría en nuestro país.

Finalmente consideramos que la falta de presupuesto también evidencia falta de interés. Si no hay un presupuesto importante, no hay un interés importante. Los gobernantes pueden pensar que como los efectos devastadores de este cambio climático no son inmediatos, no son urgentes. Y lo que gobierna es la política de la urgencia.

Además, algunas autoridades pueden pensar que como somos un país pobre y existiendo problemas en el sector salud y seguridad, no podemos esperar que exista un cambio significativo en el porcentaje que actualmente se invierte en las áreas vinculadas a la lucha contra el cambio climático en general y en particular en favor de la transición energética hacia las energías renovables. Al respecto discrepamos de esa falta de interés disfrazada de falta de importancia, pues consideramos que se puede no sólo aumentar sino mejorar la gestión, 
haciendo entender que el tema climático es transversal a todas las actividades públicas y privadas, y entendiendo que con poco presupuesto se pueden lograr grandes resultados.

La resistencia al cambio y la dificultad para adaptarse a las nuevas tecnologías por parte de la población es un fenómeno natural y lo entendemos también como desidia, hasta que los beneficios económicos de la adopción de dichas medidas sean más evidentes a nivel micro y a corto plazo o hasta que las sanciones por el incumplimiento de las normas sean efectivamente perseguidas y sancionadas.

En cuanto al problema de la resistencia al cambio que se atribuye a la sociedad civil, se podría afirmar que es corresponsable, como se puede apreciar en el mantenimiento de los malos hábitos de consumo y de manejo de residuos.

En cuanto a la corrupción, la corrupción existe independientemente del conocimiento de la importancia de la lucha contra el cambio climático o de que existan las condiciones jurídicas y financieras para obtener réditos económicos y políticos en el corto plazo. Lo que hace la corrupción es ahuyentar la inversión privada o encarecerla, porque los hace incurrir en sobrecostos y porque eleva el riesgo de la inversión al incurrirse en el delito de cohecho. Así, el mecanismo de obras por impuestos, entre otros, no tiene el avance que debería tener porque los empresarios no encuentran atractivo este tipo de inversiones por el sistema de corrupción enraizado en nuestro país.

Por otro lado, el tema de corrupción influye en tanto que las decisiones y recursos son guiados por el interés particular de corto plazo antes que por el bienestar general, que suele ser de mediano o largo plazo. 


\section{Posibles soluciones}

Cuando se abordaron las soluciones para cada una de las limitaciones, cada grupo concluyó en lo siguiente:

Tabla 2: Propuestas de los gobiernos subnacionales para implementar el Acuerdo de París

\begin{tabular}{|c|c|}
\hline Primer grupo & $\begin{array}{l}\text { - Como política de Estado, debería establecerse una institución } \\
\text { capacitadora de actividades municipales y regionales. } \\
\text { - Hacer un plan de desarrollo orientado al manejo ambiental. } \\
\text { - Reclutar profesionales competentes para la gestión de las } \\
\text { actividades ambientales. }\end{array}$ \\
\hline Segundo grupo & $\begin{array}{l}\text { - Trabajar en una currícula atractiva en las universidades. } \\
\text { - Mayor difusión. } \\
\text { - Incentivos en viviendas verdes. } \\
\text { - Planes de urbanización verdes. } \\
\text { - Créditos favorables para proyectos e inversiones verdes. } \\
\text { - Planeamiento de la vocación profesional a mediano y largo } \\
\text { plazo. }\end{array}$ \\
\hline Tercer grupo & $\begin{array}{l}\text { - Implementación de medidas con carácter obligatorio para los } \\
\text { funcionarios responsables, autoridades y sociedad civil. } \\
\text { - Incentivar espacios de investigación, disertación y difusión } \\
\text { de oportunidades laborales que fomenten el interés de } \\
\text { futuros profesionales. } \\
\text { - Crear equipos descentralizados para difundir la información } \\
\text { en las municipalidades mediantes charlas, talleres y cursos. }\end{array}$ \\
\hline Cuarto grupo & $\begin{array}{l}\text { - Implementación de un programa de educación ambiental. } \\
\text { - Tener técnicos capacitados con conocimiento en gestión } \\
\text { ambiental. } \\
\text { - Crear un organismo fiscalizador. } \\
\text { - Incentivos económicos y tributarios. } \\
\text { - Líneas de financiamiento de bajo costo. }\end{array}$ \\
\hline
\end{tabular}

En cuanto a la problemática de la ignorancia, coinciden en que ésta se debe combatir entre otras medidas mediante el establecimiento de una institución capacitadora de actividades municipales y regionales; el reclutamiento de técnicos y profesionales competentes para la gestión de las actividades ambientales; trabajar una currícula atractiva en las universidades; planeamiento de la vocación profesional a mediano y largo plazo; incentivar espacios de investigación, disertación y difusión de oportunidades laborales que fomenten el interés de futuros profesionales; crear equipos descentralizados para difundir la información en las municipalidades mediante charlas, talleres y cursos; así como una mayor difusión de las normas, incentivos, financiamiento y tecnologías. 
Al respecto podríamos adicionar que la atención se puede también enfocar en los colegios, y en la educación de los educadores; se pueden hacer en este campo iniciativas de gran impacto.

En cuanto a la desidia, se propuso el incentivo de viviendas verdes; planes de urbanización verde; créditos favorables para proyectos e inversiones verdes; incentivos económicos y tributarios; así como líneas de financiamiento de bajo costo. Asimismo. consideramos que, dentro de las propuestas expresadas en el taller, aquellas que plantean la necesidad de hacer un plan de desarrollo orientado al manejo ambiental también combaten la falta de interés, siempre que haya un seguimiento posterior y un incentivo o una sanción por resultados.

Por otro lado, consideramos que si bien las normas reglamentarias de la generación distribuida en nuestro país, así como el reglamento de ley del Cambio Climático, las normas que otorgan potencia firme a las fuentes de energías renovables no convencionales y aquellas que promueven el parque automotor eléctrico, van a generar un aceleramiento de la transición energética y coadyuvarán al objetivo del Acuerdo de París, para una mayor velocidad es necesaria la dación de normas tributarias y administrativas no sólo nacionales sino también regionales y municipales.

En cuanto a la corrupción, los integrantes del taller opinaron que se debe combatir mediante la implementación de medidas con carácter obligatorio para los funcionarios responsables, autoridades y sociedad civil, así como crear un organismo fiscalizador.

Al respecto consideramos que la corrupción se expande con el mal ejemplo, la impunidad y la falta de presupuesto para crear equipos de fiscalización y control. Esta ha inmovilizado y encarecido a la inversión privada pero las acciones y resultados anticorrupción en nuestro país permiten vislumbrar un nuevo patrón ético no sólo a nivel gubernamental sino también empresarial.

Por tanto, se necesita combatir la impunidad mediante la aplicación de controles suficientes, financiados y sanciones efectivas para evitarla.

La corrupción es un patrón heredado, una costumbre, un mal hábito que puede y se está combatiendo con las medidas de transparencia, control y sanción en la gestión pública y privada que se vienen realizando en nuestro país.

Así, el Organismo de Evaluación y Fiscalización Ambiental (OEFA) y los juzgados ambientales que se vienen implementando están combatiendo la contaminación ambiental, que se da por las emisiones de gases tóxicos, vertimientos contaminantes en la atmósfera, el suelo, etc., con presupuestos que debieran ser aumentados para un mayor seguimiento y mejor ejecución de sanciones, entre otras medidas que también podrían ser implementadas a nivel subnacional. 


\section{Conclusiones}

Los gobiernos azules están creciendo en nuestro país gracias a las iniciativas que viene liderando el gobierno central en materia de energía, cambio climático, electromovilidad, manejo de basura, del plástico y lucha contra la contaminación, que están siendo replicadas en algunos gobiernos municipales.

Sin embargo, de este Foro-taller se desprende que la ignorancia, la desidia y la corrupción están presente como los principales obstáculos para impulsar las energías renovables y el Acuerdo de París.

Asimismo, este Foro-taller nos señala que a pesar de lo claro del riesgo para nuestra sociedad y del compromiso asumido por nuestro país frente al cambio climático, hay mucho trabajo por hacer para que no sea solo el Estado, sino que sea toda la nación la que se involucre en una gesta que trascienda nuestros miedos y ambiciones, para que se concreten nuestros sueños y proyectos.

Se desprende de este trabajo que debemos trabajar no solo de arriba hacia abajo, sino de abajo hacia arriba. Hay que unirse para hacer protagonistas y no solo espectadores a los gobiernos locales y regionales, a la empresa privada y a la sociedad civil en general.

Y, para ello, se precisa que el Estado perciba a la sociedad civil y a la empresa privada como aliados estratégicos para un combate eficaz y necesario contra el cambio climático. Es un enfoque que proviene de la realidad, de la experiencia, aquella que dice que, cuando la sociedad civil y las empresas encuentran un beneficio al ayudar a los demás, la sociedad en su conjunto prospera.

Es la comunión del interés general y del particular la que logrará el ansiado equilibrio indispensable no solo para sobrevivir, sino para obtener aquella sociedad de bienestar que algunos, como Tomás Moro o Platón, vislumbraron como deseable, necesaria y posible. 